\title{
Modern technology assists disabled competitors: the first "Cybathlon" special competition in Zürich
}

\author{
WŁODZIMIERZ S. ERDMANN ${ }^{1 *}$, PIOTR ASCHENBRENNER ${ }^{1}$, \\ VASILIOS GIOVANIS ${ }^{2}$ \\ ${ }^{1}$ Faculty of Physical Culture, Gdansk University of Physical Education and Sport, Gdańsk, Poland. \\ ${ }^{2}$ School of Physical Education and Sport Science, National and Kapodistrian University of Athens, Dafni, Athens, Greece.
}

\begin{abstract}
Purpose: The purpose of the study was presentation of modern bioengineering technology in order to help people with severe disabilities. Methods: Bioengineering industry can offer severely disabled people several devices in order to enable them to take part in the competition different than Paralympics. The first international competition for people with disabilities supported by modern assistive technology, such as sensors, motors, displays were allowed to compete in Cybathlon held in Zürich in 2016. About 70 athletes and their teams from 25 countries appeared at the event. Results: There were six disciplines (races): 1) Powered Arms (Upper Extremities) Prostheses Race, 2) Powered Legs (Lower Extremities) Prostheses Race, 3) Powered Wheelchair Race, 4) Powered Exoskeleton Race, 5) Functional Electrical Stimulation Bike Race, 6) Brain-Computer Interface Race. About a quarter of the teams represented industry and the rest represented university laboratories. Conclusions: The competition was a success. The organisers have decided for it to be organized every four years, just like the Olympic Games for able bodied competitors. The main inventor of the event professor Robert Riener from Zürich Polytechnic (ETHZ) said assistive technology should: a) be user-friendly b) to function well, c) be affordable, d) to be used within the barrier-free environment.
\end{abstract}

Key words: disabled athletes, assistive technology, competition, Cybathlon

\section{Introduction}

Rehabilitation through physiotherapy, especially kinesiotherapy, acts to bring people with disabilities back into society. It is very emotional when patients who previously could not leave their beds can sit, stand, and walk after several months of intense training. These patients are also instructed to do exercises by themselves in order to enhance their activity [1]. In addition, technology, including robotics, helps people with disabilities in their everyday lives [2]. Every contemporary sport uses some kind of technical support providing the possibility to compete (e.g., balls, bikes), to enhance performance (e.g., aerodynamic covers), and to protect the body (e.g., helmets). These devices are also used by disabled sportspeople. In addition, some special equipment is utilised only by them [3], [4].

Sometimes, there are controversies about whether particular equipment can be used. Much is done to provide an equal chance to all the participants in a sport event, not only by establishing many divisions and classes for participants with different disabilities, but also when disabled sportspersons want to compete with able-bodied competitors.

The bioengineering industry develops special technologies for disabled sportspeople who are not allowed to participate at the Paralympics. It is difficult to achieve equal chances within the same class of competition. Several laboratories are working to design equipment with better mechanical characteristics (usually, this equipment is personalised) in order for elite adapted sportspeople to obtain better results compared to their

\footnotetext{
* Corresponding author: Włodzimierz S. Erdmann, Faculty of Physical Culture, Gdansk University of Physical Education and Sport, ul. Kazimierza Górskiego 1, 80-336 Gdańsk, Poland. Phone: +48605304939, e-mail: wlodzimierz.erdmann@awf.gda.pl

Received: January 9th, 2020

Accepted for publication: May 22nd, 2020
} 
opponents [5]. In Zürich, a new kind of competition was organised, namely the "Cybathlon". This was a championship where people with physical disabilities competed against each other at the tasks of daily life with the aid of advanced assistive devices, including robotic technologies [6].

The process of introducing a new device to the public is long. It consists mainly of: 1) proposing an idea, 2) elaboration of a project, 3 ) making a model prototype, 4) making a real dimensions device, 5) checking an application of the device, 6) starting industry manufacturing. The "Cybathlon" competition played a role of checking an application of different types devices.

\section{Materials and methods}

\subsection{Participants}

About 70 athletes and their teams from about 50 companies and universities from 25 countries (from all continents) appeared at the event. About a quarter of the teams represented industry, and the rest represented university laboratories. People with disabilities that prevent them from taking part in the Paralympics, being supported by modern assistive technology such as sensors, motors, and displays, were allowed to compete.

\subsection{Disciplines}

There were six disciplines during the event: 1) Powered Arms (Upper Extremities) Prostheses Race, 2) Powered Legs (Lower Extremities) Prostheses Race, 3) Functional Electrical Stimulation Bike Race 4) Powered Wheelchair Race, 5) Powered Exoskeleton Race, 6) Brain-Computer Interface Race. The tasks were chosen not from sports for able-bodied competitors but rather from everyday living tasks, such as walking on uneven surfaces, stair climbing, opening and closing doors, and other tasks.

\subsection{Location and time of the competition}

"Cybathlon", the first international competition for people with disabilities supported by modern assistive technology, took place in Zürich, Switzerland. The Swiss Arena Kloten was the venue for "Cybathlon" (Fig. 1). The competition took place on October 8, 2016 from 10 am to $6 \mathrm{pm}$.

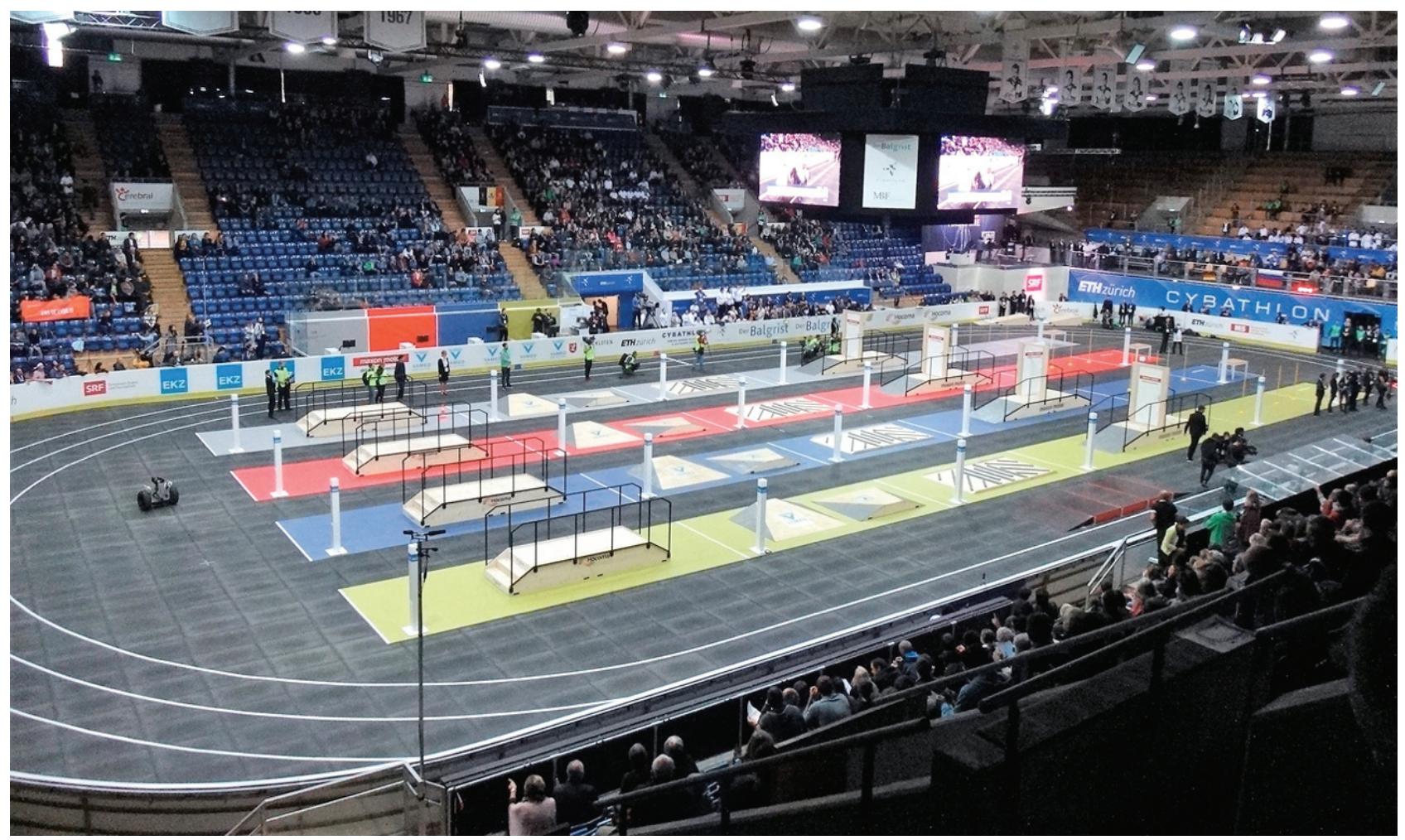

Fig. 1. General view of the „Cybathlon” venue in Zürich 


\section{Results}

\subsection{Powered arms (upper extremities) prostheses race}

Pilots with one or both upper extremities amputated above or below the elbow competed in the race using prosthetics handled with different kinds of controllers (Fig. 2a). The pilots executed various steps and movements using motorised prostheses. The tasks were as follows [7]:

a) Puzzle. Placing all the puzzle pieces onto a neighbouring table; challenge: fine motor skills, multiple types of grips, finger strength.

b) Wire Loop. Moving a wire loop with a grip along a curved wire without touching it; challenge: freedom of movement in the forearm and wrist, precision.
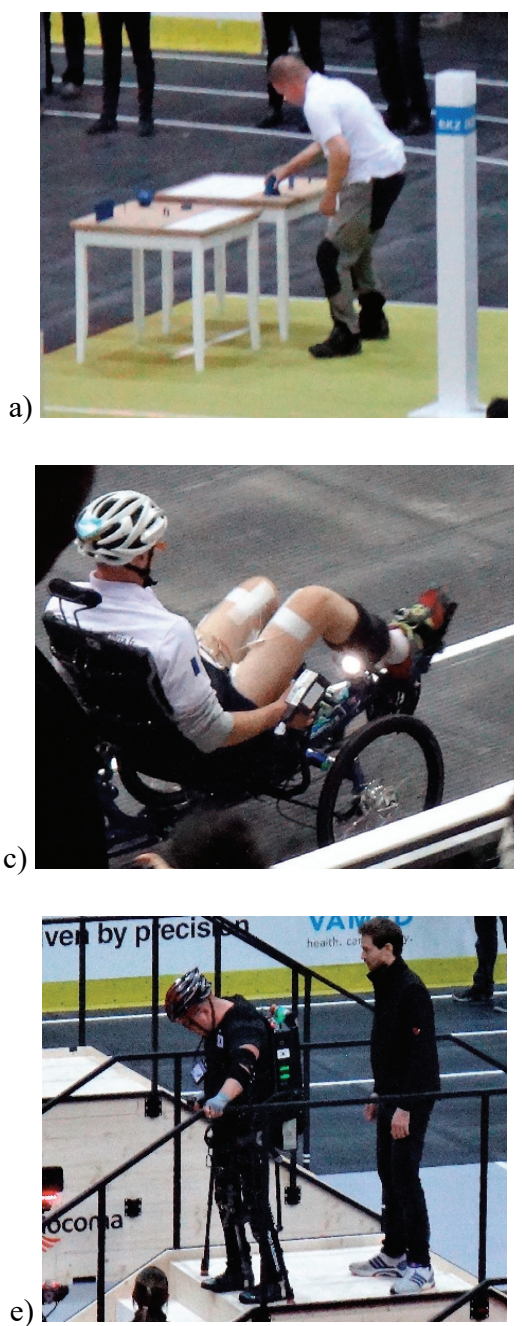

c) Setting the Table. Bringing objects from a rack to a table, opening and closing a door, inserting a blue light bulb into a lamp; challenge: fine motor skills, multiple types of grips, balancing objects on a tray, coordination of both arms.

d) Preparing Breakfest. Opening a can, a bottle, and a jar, unwrapping a sugar cube, cutting a slice of bread; challenge: hand strength in opening the can, the jar, and the bottle, strength for slicing the bread, fine motor skills.

e) Hanging Up Laundry. Hanging a t-shirt on a line with two clothes pegs, buttoning a blazer and zipping up a jacket, putting them on hangers; challenge: fine motor skills, coordination of both hands.

f) Carrying Objects. Moving all the objects via the stairs onto a table; challenge: stable connection between the prostheses' socket and the arm stump, hand strength.
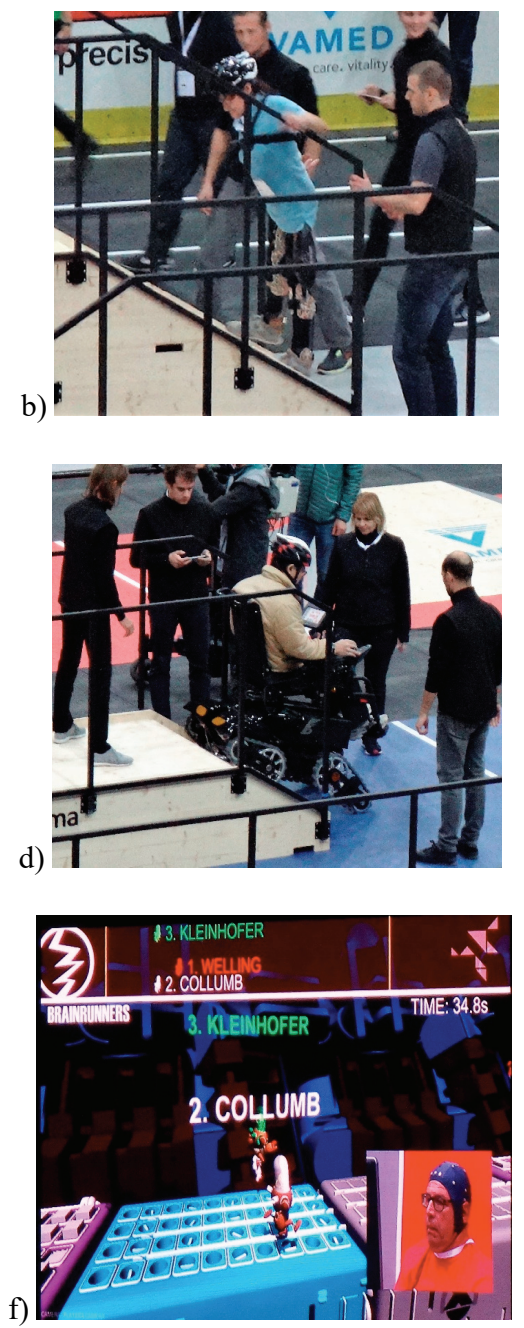

Fig. 2. „Cybathlon” disciplines (races): a) upper extremity prosthesis, b) lower extremity prosthesis, c) functional electro-stimulation bike, d) powered wheelchair (here competitor rides up-stairs being with his back towards the direction of a ride), e) exoskeleton, f) brain - computer interface. For wheelchair and exoskeleton users, few guards always assisted the competitor during the race 


\subsection{Powered leg (lower extremities) prostheses race}

The pilots executed various steps and movements using motorised prostheses (Fig. 2b). The tasks were as follows [7]:

a) Sofa. Sitting down and standing up five times; challenge: lower extremity strength, power supply.

b) Hurdles. Passing the hurdles without knocking them down; challenge: ability to bend and control the movements of the knee joint.

c) Ramp \& Door. Climbing a steep ramp, opening and closing a door, going down the other side; challenge: ability to flex the knee and the ankle joints, bending stability and motor power at the ramp.

d) Stones. Stepping onto each stone with one foot; challenge: precision and balancing on one foot while stepping to the next stone.

e) Tilted Path. Walking over ramps; challenge: inversion/eversion of the ankle joint.

f) Stairs. Walking over the stairs three times while balancing a cup on a saucer and apples on a plate; placing only one foot alternatively on each step; challenge: ability to bend the knee joint, strength, precision, stability.

\subsection{Functional electrical stimulation bike race}

Pilots with complete paraplegia were allowed to compete in the race. Their motor nerves were artificially stimulated in order to achieve muscle contraction. This was used to push the pedals of the bike. Stimulating electrodes were placed on the skin (they could be also implanted) and an applied current caused the contraction of the muscles. The pilots controlled the power of the stimulation by themselves. Only pilots with complete spinal cord injuries were allowed to participate using non-motorised bikes (Fig. 2c). Before the race, the pilots trained in order to obtain enough muscle endurance. They regulated the velocity of the bike in relation to their endurance. Two pilots started at the same time and competed at the race along a circular track. The challenge was to skilfully stimulate the muscles so that they achieved a high velocity without becoming fatigued too quickly. The time limit was eight minutes [7].

Hereby, the best way to compete was to ride a little bit slower than average at the start fragment (as a kind of warm-up, despite the warm-up before the race), to maintain a steady velocity during the race, and the velocity could be a little bit higher than average at the end. In addition, deviations from the velocity trend line should have been minimised [8].

\subsection{Powered wheelchair race}

The pilots competed on a track using motorised wheelchairs, overcoming obstacles that are typical in daily life. Motorised wheelchairs controlled by a joystick, touchpad or similar technology were allowed in the competition (Fig. 2d). The organisers wanted to encourage the teams to overcome obstacles such as narrow doorways, uneven pavement, steep slopes and other problems. The tasks for the competitors were as follows [7]:

a) Table. Driving up to a table, placing the thighs halfway underneath it without moving the table; challenge: size of the wheelchair.

b) Slalom. Passing between the slalom poles without displacing them; challenge: size of the wheelchair, precise maneuvering.

c) Ramp \& Door. Climbing a steep ramp, opening and closing a door, going down the other side; challenge: reaching down and pressing the door handle, maneuvering in a confined space.

d) Rough terrain. Driving over the terrain; challenge: gripping and performance of the wheels.

e) Tilted Path. Driving over the terrain; challenge: drift and tipping stability, power.

f) Stairs. Driving over one time; challenge: ability to climb stairs, power.

\subsection{Powered exoskeleton race}

Pilots with complete paraplegia resulting from spinal cord injuries negotiated an obstacle course consisting of typical everyday tasks. Exoskeletons are wearable, motorised supports (orthoses) which allow people to do at least a few general tasks they did when they were healthy. People who were lying on a bed or sitting on a chair now can walk independently. The pilots were asked to guide their exoskeleton to correctly perform the movements (Fig. 2e). The tasks for the competitors were as follows [7]:

a) Sofa. Sitting down and standing up one time; challenge: range of motion in the knee and hip joints, strength.

b) Slalom. Passing between the slalom poles without displacing them; challenge: precision, agility.

c) Ramp \& Door. Climbing a steep ramp, opening and closing a door, going down the other side; chal- 
lenge: flexibility of the foot, knee, and hip joints, stability when opening the door.

d) Stones. Stepping onto each stone with at least one foot, and crutches could be placed beside the stones; challenge: adapting step lengths and widths, precision.

e) Tilted Path. Walking over ramps; challenge: flexibility of the foot joints, stability.

e) Stairs. Going up and down one time, and each step had to be stepped on by at least one foot; challenge: ranges of the knee and hip joints, strength, precision.

\subsection{Brain-computer interface race}

The pilots used brain-computer interfaces (BCI) in the form of a cap with electrodes put on the head to control avatars in a specially developed computer game (Fig. 2f). BCIs can detect brain signals with the help of electroencephalography (EEG). The participants had complete or severe loss of motor function (paralysis) from the neck down due to a spinal cord injury (SCI), stroke, neurological disease, or another trauma. There were 16 pads (500 virtual meters). The challenge was for the avatar to move correctly and quickly only if the computer received the correct brain signal at a specific moment. Otherwise, the avatar were to slow down. There were the following commands: 1) Rotate - the avatar rotated (danced) over the playing pads (blue), 2) Jump - the avatar jumped over spikes (purple), 3) Slide - the avatar slid under "dangerous rays" (yellow), 4) No input - on the neutral fields (gray), the pilot was asked "to think nothing" to prevent the avatar from slowing down. The time limit was four minutes [7].

\section{Discussion}

\subsection{General comments on the "Cybathlon"}

The tasks for the upper and lower extremities were accomplished with great accuracy and with significant speed by all the participants. Also, the FES bike race was done by almost all the competitors with good times. During the other competitions, there were difficulties during the races. Some participants using exoskeletons and wheelchairs had problems, especially in climbing stairs. Not all competitors finished their races.
The exoskeletons and wheelchairs were usually prototypes, so further technical improvement is needed. Also, the prototypes are usually initially tested on nondisabled subjects. They can compensate by maintaining their balance using several muscles. On the other hand, when a paralysed person uses an exoskeleton, the machine needs to bear all their weight although the person also uses crutches, which are mostly for maintaining balance [9]. Exoskeletons are still in their early stages of construction, so more tests involving disabled people are needed. Wheelchairs that are powered electrically usually have both wheels and caterpillar technology. As a device with a computer on board, it must have both proper construction and proper software. The companies which develop their devices also provide the money for their production. University labs must make efforts to obtain scientific grants or find sponsors. For example, the Zürich Polytechnic students who built their wheelchair using caterpillar technology contacted 37 sponsors [10].

"Cybathlon" was a kind of competition where both the athletes and the manufacturers that produced the competition devices were very valuable in obtaining the final result. This is similar to the situation in motor sports (automobile, motorbike), but in the "Cybathlon", the role of the manufacturers is currently even more important.

\subsection{Improvement of the competition}

The rules for these types of competitions must more closely resemble the rules of sport disciplines for ablebodied competitions. For example, during the FES Bike Race, the rules stated: 1) The first to reach the finish line is the winner (5 laps, 750 meters), and 2) the pilot who starts in the outside lane could change to the inside lane after half a lap, as long as he/she does not endanger the other pilot [7]. During the 800 $\mathrm{m}$ race in athletics, the competitors start at different starting lines and are separated in their own lanes, but after running $100 \mathrm{~m}$, i.e., until the end of the first bend, they change their lanes, moving towards the innermost lane. The FES Bike Race competitors also started from different starting lines at the elevation of a ramp (Fig. 3). The outside ramp was positioned at about $2.5 \mathrm{~m}$ to the front. But the difference in the length of the inside and outside tracks was much greater. Assuming that the radius of the inside track (r.i) $=10.8 \mathrm{~m}$ and of the outside track (r.o) $=12.0 \mathrm{~m}$, the difference in the length of the curve was about $7.5 \mathrm{~m}$, i.e., three times longer than the starting difference of the competitors' positioning. Another possi- 


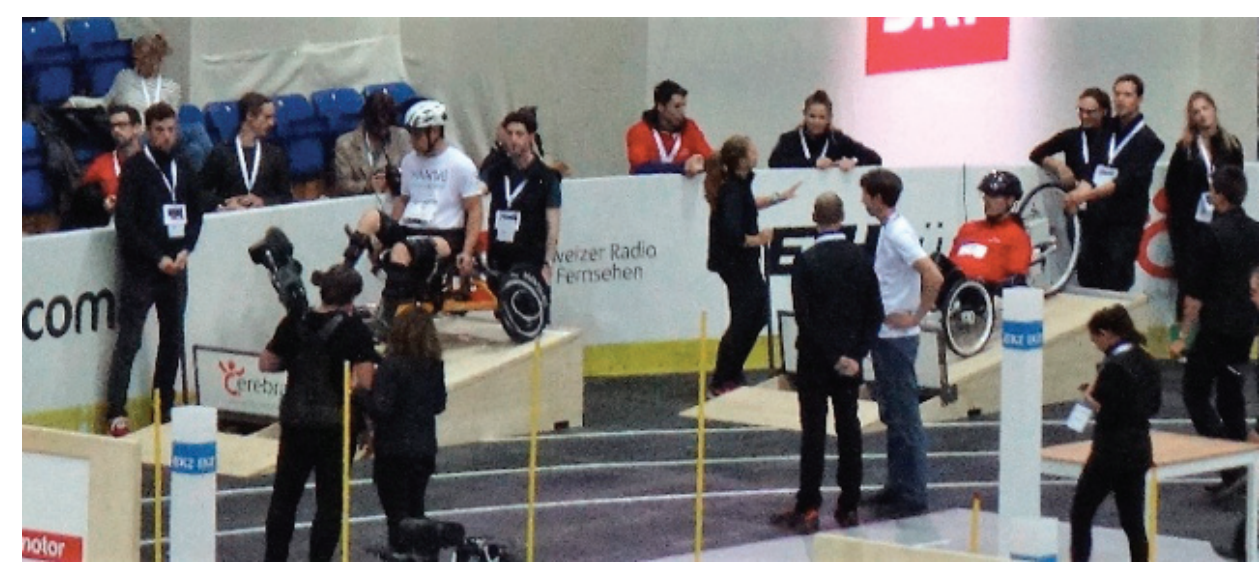

Fig. 3. Start of FES bikes from the ramps

bility is to ride like 400-metre runners run in athletics, i.e., without changing lanes, or riding like indoor bicycle riders, i.e., starting from the opposite lines of a track.

Another improvement would be the regulation of the obstacles that had to be overcome and the regulation of the objects that needed to be operated. There are two reasons for this. First, if another institution wants to organise the same competition, it would be better to use the same facility and its equipment. The second problem is training. In sport disciplines for able-bodied competitors, all the facilities and equipment are the same for every competition. Thus, the competitors can train at home in the same surroundings they would find during a competition in another place.

\subsection{Parallel events}

Along with the competition, there were also parallel events: 1) Hands-on demos; here, the spectators could experience how it feels to play a computer game with their thoughts, to grasp objects with an artificial hand (Fig. 4a), to walk with an artificial lower extremity (Fig. 4b), to struggle with uneven terrain in a wheelchair; 2) From Captain Hook to Iron Man; this was an exhibition from the beginning of assistive aids up to modern times; 3) "Let's fätz" - Let's get moving; this was an outdoor sports programme for both children and adults; 4) Flight Simulation "Symbiotic Drone"; visitors could fly like a drone using virtual reality goggles and a special jacket that records body movements [7].

\section{Conclusions}

The main creator of the event, Professor Robert Riener from Zürich Polytechnic (ETH), said that assistive technology should: a) be user-friendly; b) function well; c) be affordable; and d) be used within a barrierfree environment. Professor Riener anticipates the further development of assistive technology. For

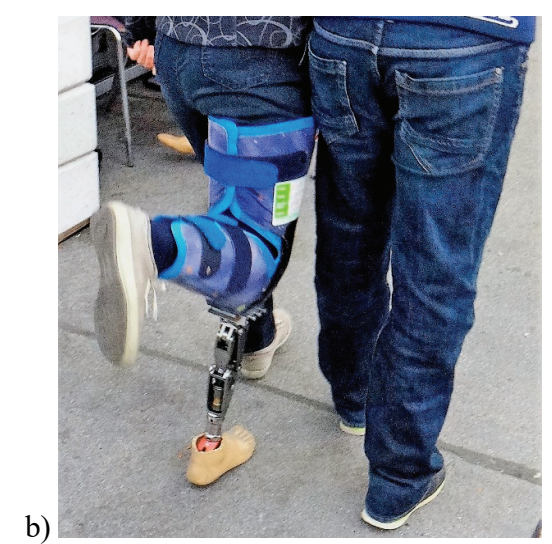

b) a)

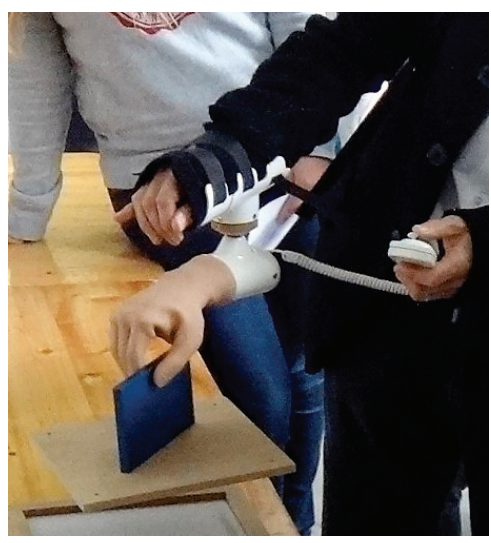

Fig. 4. During „Cybathlon” spectators could try devices for disabled people:

a) artificial hand, b) artificial lower leg 
example, exoskeletons will be integrated with clothing [11].

The competition was a success. It was decided for it to be organised every four years. The next "Cybathlon" championship for robot-assisted competitors with disabilities is to take place in 2020. It should conform more to typical sport competitions in order to provide equal chances for all the participating teams to the greatest extent possible.

\section{References}

[1] NoOiJen C.F.J., Stam H.J., Bergen M.P., Bongers-JansSen H.M.H., Valent L., VAn Langevelt S. et al., $A$ behavioural intervention increases physical activity in people with subacute spinal cord injury: a randomised trial, J. Physioth., 2016, 62, 35-41.

[2] RIENER R., Rehabilitation robotics. Foundations and Trends in Robotics. Vol. 3(1-2), 1-137, now Publishers, BostonDelft 2012.

[3] ERDMANN W.S., Technical development and its influence on Olympic sport (in Polish), [in:] J. Czerwinski, H. Sozanski (Eds.), Contemporary Olympic Sport. An outline of the prob- lems, Gdansk University of Physical Education and Sport, Gdansk 2009, 227-253.

[4] ERDMANN W.S., Technical devices and modern technologies within sport, (in Polish), [in:] H. Sozanski, J. Czerwinski, J. Sadowski (Eds.), Fundamentals of theory and technology of sport training, J. Piłsudski University of Physical Education, Warsaw-Biala Podlaska 2013, 207-227.

[5] ERDMANN W.S., Engineering of movement rehabilitation. An outline, (in Polish). Gdansk University of Technology, 2016.

[6] RIENER R., The Cybathlon promotes the development of assistive technology for people with physical disabilities, J. NeuroEng. Rehab., 2016, 13 (49), DOI: 10.1186/s12984-016-0157-2.

[7] KASIElKe N. (ed.), Programm/Programme. Cybathlon, 8. Oktober 2016, Swiss Arena Kloten, Swiss Federal Polytechnic, Zurich 2016.

[8] ERDMANN W.S., LiPINSKA P., Kinematics of marathon running tactics, Hum. Mov. Sci., 2013, Vol. 32, 1379-1392.

[9] VARILEG Ch., Learning to Walk Again, N. Kasielke (Ed.), Programm/Programme. Cybathlon, 8. Oktober 2016, Swiss Arena Kloten, Swiss Federal Polytechnic, Zurich 2016, 41-43.

[10] Scewo CH., Super Stair-Climbers, [in:] N. Kasielke (Ed.), Programm/Programme. Cybathlon, 8. Oktober 2016, Swiss Arena Kloten, Swiss Federal Polytechnic, Zurich 2016, 44- 47.

[11] RIENER R., Technology for Everyday Life, [in:] N. Kasielke (Ed.), Programm/Programme. Cybathlon, 8. Oktober 2016, Swiss Arena Kloten. Swiss Federal Polytechnic, Zurich 2016, 5-7. 